\title{
Autonomous Power Station Based on Rotary- Vane Engine with an External Supply of Heat
}

\author{
Mikhail Andreev, Yuriy Zhuravlev, Yuriy Lukyanov, Leonid Perminov \\ Pskov State University, Faculty of Electrical Engineering, Department of Drive and \\ automation systems. Address: 180760, Russia, Pskov, Lenin Square 2
}

\begin{abstract}
Rotary-vane engine (RVE) with an external supply of heat is an aggregate consisting of two modules with a common output shaft, the heating device (heater) of working medium and the cooling device (cooler) of working medium, which connected with inlet and outlet ports of modules by system of pipeworks. Each module has two rotors with two vanes on each. Between the corresponding plane surfaces of the four vanes four working volumes are formed wherein thermodynamic cycle steps: ingress, compressing, heat intake, expansion stroke, discharge, outward heat transmission are going simultaneously. The angular displacement of modules relative to one another occurs pumping the working medium through the heater and cooler, which allows the conversion of thermal energy into mechanical work. Design features of the RVE with an external supply of heat allows create a closed gas-vapor cycle.

The main specified advantages of the RVE with an external supply of heat are: fewer noxious emissions, multifuel capability, high motor potential (service life). Different problems of creation external combustion engines such as structural complexity of construction units, absence of adequate mathematical model of designed RVE with an external supply of heat are also pointed. The construction of the RVE with an external supply of heat developed in Pskov Polytechnic Institute (now the Pskov State University), the operation concept of the engine, the physical processes in the chamber modules RVE with an external supply of heat during each step and the mathematical model describing the physical processes proceeding in chamber RVE with an external supply of heat modules are considered.
\end{abstract}

Keywords - internal combustion engine, rotary-vane engine, autonomous power station based on RVE.

\section{INTRODUCTION}

The development and application of internal combustion engines (ICE) are now acquired comprehensive. Numerous research and development engine have made into a very complex and at the same time, reliable and universal system. However, experience in long-term operation in the vehicle identified weaknesses that are nearly impossible to exclude by upgrading the design of the engine without affecting the basic principles of its construction, such as mechanical friction and the process of internal combustion.

The main drawback of the ICE, which is a result of the mass distribution of automobile transport has taken the lead, became a factor in environmental pollution by exhaust gases. The share of pollutants released into the air from the exhaust gases of automobile engines, up to $63 \%$ of the total pollution. Therefore, in recent decades, the world's stricter requirements for environmental standards for vehicles, and in particular this applies to internal combustion engines. [1] ICE, consuming a fifth of primary energy [2], are a major source of pollution in the immediate area of human breathing. [3] However, the planned measures, even if their full implementation, can only reduce the rate of increase of the polluting engines, especially vehicles, by the rapid growth of the number and capacity.

Thus, there is a need of a fundamentally different production engine capable to change the situation, working on different types of fuel and with no hazardous emissions. In this regard, one can submit the following requirements for modern engines:

- Reduce the amount of toxic emissions

- Reduce the release of heat into the atmosphere

- Reduce metal consumption engine

- Reduce Noise and Vibration

- Use for any type of motor fuels, including hydrogen

- Use renewable energy sources.

\section{ENGINES WITH AN EXTERNAL SUPPLY OF HEAT}

By ecological use of any type of fuel, the engine has the best characteristics with an external supply of heat realizing the Stirling cycle. External supply of heat allows to apply different heat sources, without any substantive changes engine design. Practically all fossil fuels from solid to gaseous can be used in such engines.

Consider the main advantages of engines with an external supply of heat (external combustion engine).

Thermal coefficient of efficiency is the efficiency of the Carnot cycle and considering temperature achieved at now is about $60 \%$. In external combustion engine power is controlled by changing the pressure of working medium in the inner cavities of the engine. At the same time, the temperature of the heater and cooler automatically maintained constant. Therefore, when the power and speed of the shaft boundary temperatures and their quotient remain constant. Through this engine efficiency when operating at Unrated mode varies only slightly. External 
combustion engine, having high-pressure working substance in internal cavities and sufficiently high temperature heater tubes, easy starts at any ambient temperature. It depends solely on the starting reliability, which can be ignited fuel in the combustion chamber.

Since the external combustion engine is fully sealed, dust, falling into the air charge the combustion chamber from the environment, does not fall into the cylinders and crankcase. As a consequence, there is no additional abrasive wear of moving parts drive mechanism.

Speed limit offensive creep of a material heater parts operating at high temperature determines external combustion engine motor resource. In general, any external combustion engine can be guaranteed to withstand brief 50-80\%-ing the overload without significant reduction in longevity, as the temperature of parts remains unchanged.

External combustion is continuous and does not have an explosive character, so the combustion and exhaust noise is almost no generated. In addition, the pressure in the cylinder external combustion engine changes smoothly, almost sinusoidally. Inlet and outlet valves, and valve gear is missing. The noise level of the engine by an average of 20-30 dB lower than that of diesel the same power.

Based on the above can say that in the field of engines had technical contradiction: on the one hand we have a compact and low-cost internal combustion engines, on the other hand, massive and expensive to manufacture engines with an external supply of heat.

\section{PROBLEMS IN CREATING ENGINES WITH AN EXTERNAL SUPPLY OF HEAT}

The shortcomings plunger Stirling engine. The first is the complexity of the case design of individual units, problems with the seals, power control, etc. Features of embodiment conditioned by employed working medium. So, for example, helium has superfluidity, which places greater demands on the sealing elements of the working plunger, etc. Second formation of promising image, intended for production Stirling machines is impossible without the development of new technical solutions to the major units. The third problem - is a high level of production technology.

This problem needs to be used in machines Stirling heat resistant alloys and nonferrous metals, their welding and soldering. A separate question - making the regenerator and attachments for it to provide on the one hand a high heat capacity, and on the other hand - a low hydraulic resistance. All this requires highly skilled manpower and modern technological equipment.

The main problems associated with the creation of highly effective plunger Stirling machines and constraints still be widely used in various fields of technology are, above all, the creation of adequate mathematical models of a machine Stirling and the appropriate method of calculation. The complexity of calculating the projected machine is determined by the complexity of the implementation of the Stirling thermodynamic cycle in real machines, due to nonstationarity heat-mass exchange in the inner loop, by the continuity of movement of the plunger. The lack of adequate mathematical models and calculation methods are the main causes of failure of some known foreign companies and domestic companies trying without serious scientific study, only through a rough calculation and experimental data to solve the problem of creating Stirling engines and refrigerators. Currently, how many firms and many methods of calculating Stirling machines, with the calculation methods are trade secrets.

Foreign experience of a modern high-efficiency Stirling machines shows that without accurate mathematical modeling of workflows and the optimal design the basic units finishing machines designed becomes a long-term debilitating experimental studies.

\section{ROTARY-VANE MACHINE}

In 2007, the Pskov Polytechnical Institute (now Pskov State University) won the tender under the federal program and signed a state contract № 02.516.11.6031 with the Federal Agency for Science and Innovation to prosecution of research on "Development of a mathematical model the flow thermodynamic cycle with an external supply of heat, allowing to create an environmentally clean engine rotary-vane type".

The result was the creation of methods analysis and design RVE with an external supply of heat, in particular to create a mathematical model of single engine components: the motion conversion mechanism, rotary-vane groups, and a mathematical model, which confirms possibility of implementing the thermodynamic cycle with an external supply of heat in RVE. For experimental studies have been created and studied the breadboard construction of mechanism transducer motion and breadboard construction of the combustion chamber. Obtained results were evidence base correctness of theoretical calculations. As an example, figure 1 shows the general construction of the two-module RVE with an external supply of heat.

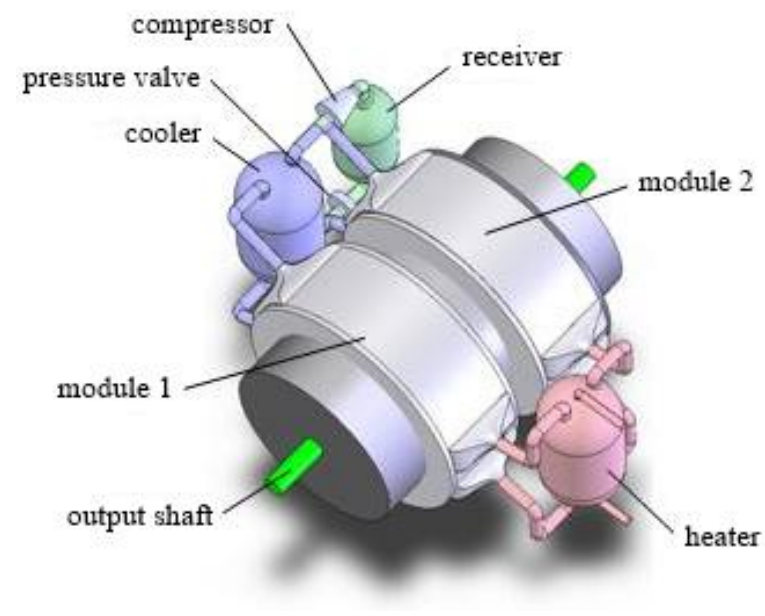

Fig. 1. RVE with an external supply of heat 


\section{THE DESIGN}

RVE with an external supply of heat consists of two working modules 1 and 2 . The modules have a common output shaft. The engine contains working medium heating device (heater) and cooling the working medium (cooler), which are related to the conduit system inlet and outlet windows modules. To control the output torque provides controlled pressure valve, receiver and compressor, which are connected to the cold pipes. Torque control on the output shaft with the temperature by changing fuel supply to the heater.

Each module includes a main cylinder the working volume (figure 2) and the motion conversion mechanism (figure 3). In the main cylinder, the working volume has two windows connected with the heater pipes, and two windows connected with cooling pipes.

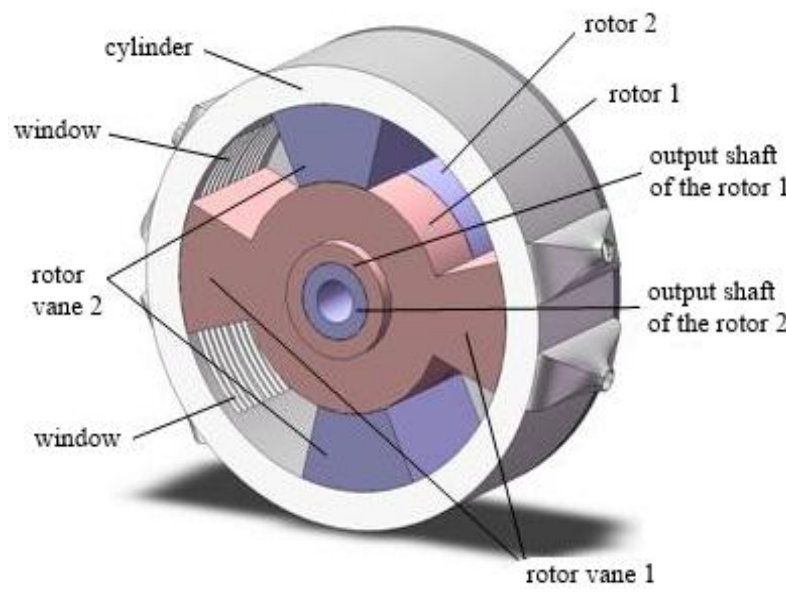

Fig. 2. Module of the RVE with an external supply of heat

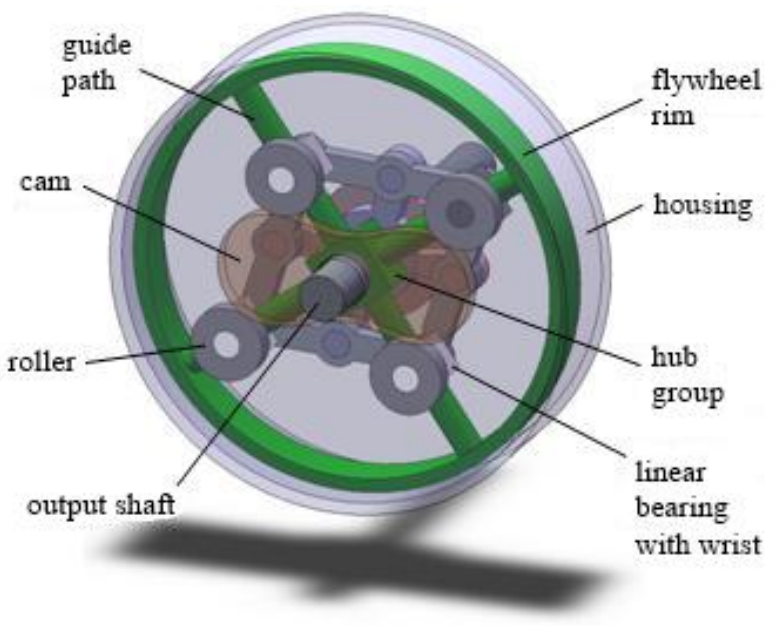

Fig. 3. Motion conversion mechanism

In the main cylinder of the working volume, energy is converted into the working medium motion of the rotor.
RVE with an external supply of heat has a cylindrical body, which are two rotor 1 and 2 with two vanes. Between the respective planes of the four vanes are formed four working volume. The output shafts of the rotor 1 and rotor 2 are hollow and mounted coaxially to each other. Inside the output shaft of the rotor 2 is the engine output shaft.

The output shafts are connected with the mechanism of the transformation movement. Engine capacity formed between the vanes rotor 1 and 2 .

Possible implementation the working volume of the cylinder in the form of spherical segments. The internal surface of each cylinder forms a hollow torus.

Motion conversion mechanism converts the vibrational-rotational rotor motion in the uniform rotation the output shaft.

Motion conversion mechanism has a housing in which the anti-friction bearings mounted engine output shaft. To the housing transmission mechanism is rigidly fixed profiled cam. On the output shaft of the motor is rigidly fixed hub group with four guide paths. On the guide paths are linear bearings with wrists that have the ability to move along the guide paths. At one end of the wrist linear bearing mounted roller which driven around on the surface of the cam.

The basis of the motion conversion mechanism is articulated four-link chain (rhomboid), consisting of four units the same length and two shafts with forks.

The ends of the rhomboid links pivotally connected to each other and are located on the wrist of a linear bearing. Shafts with forks conversion mechanism rigidly connected to the output shaft rotor. Shafts with forks have two wrists, which are inserted in the middle of the relevant parts of the rhomboid.

It should be noted that the hub with guide paths one module and the second module are rotated relative to each other at an angle of 45 degrees.

In four working volume of each module to perform simultaneous steps of the thermodynamic cycle: intake, compression, heat input, working stroke, release, heat dissipation. Due to the angular displacement modules relative to each other occurs transfer of the working fluid through the heater and cooler, which allows the conversion of thermal energy into mechanical work. Design features of the rotaryvane engine can organize a closed steam-gas cycle.

\section{POWER STATION BASED ON RVE}

Power station based on RVE can work in the long offline mode, in single mode by double overloads. If required the power station can work in parallel with the external mains. 


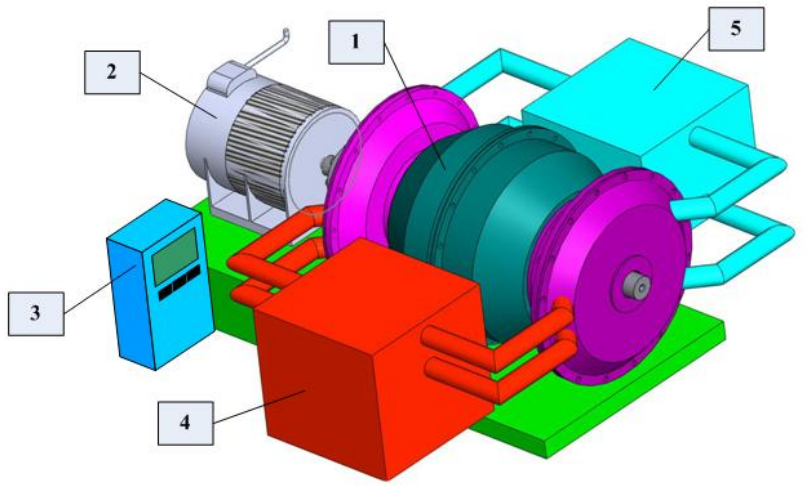

Fig. 4. Autonomous power station based on RVE with an external supply of heat

Autonomous power station based on the RVE with an external supply of heat (figure 4) consists of: twomodule rotary vane engine with an external supply of heat 1 , coaxially connected to the electric generator 2 , the control system 3 , a heater with a heat exchanger 4 and cooling with heat exchanger 5. By using different types of fuel in the design may include a device for the production of synthesis gas, batteries or capacitors, reactors for biogas, pyrolysis plant, etc.

Block diagram of the new generation power station consists of blocks of fuel preparation, storage and use of energy, including the control modes.

In accordance with the basic variants of for use different types of fuel is necessary to develop the following types of gas-generating device:
- multi-fuel gas generator, using local and renewable fuel (wood, etc.);

- multi-gas generator that uses a liquid fuel (diesel, gasoline, etc.) are available by using the existing infrastructure of filling stations.

To improve the efficiency of power stations will be used in gas generators multistage combustion, hydrogen generation initiating additives, thermochemical recuperation of exhaust gases.

\section{REFERENCES}

[1] Lukyanov Y.N., Plohov I.V., Zhuravlev Y.N. The mechanism for converting motion. Patent RF № 2374526 from 27.11.2009.

[2] Lukyanov Y.N., Plohov I.V., Zhuravlev Y.N. Rotary-vane engine with an external supply of heat. Patent RF № 2387844 from 27.04.2010.

[3] Minihanov R.N., Kogotin S.A., Degtyarev G.L., Gafurov R.A. Scientific, technical and production to ensure the development of automotive industry. Proceedings of the Second International Scientific Conference «Automobile \& technosphere». Kazan. 2001, pp. 13-19.

[4] Yuldashev A.A., Yunusov K.A., Fomin V.P. Status and prospects of the use of natural gas as a motor fuel. Proceedings of the Second International Scientific Conference «Automobile \& technosphere». Kazan. 2001, pp. 181-186.

[5] Samoilov N.P. The problem of reducing air pollution by automobile transport. Proceedings of the Second International Scientific Conference "Automobile \& technosphere». Kazan. 2001, pp. 333-334.

[6] Stirling engines. Edited by Kruglov M.G. 1977.

[7] Kirillov N.G. Stirling machines for high-performance and environmentally clean autonomous power supply systems. Chemical and Petroleum Engineering. Vol. 12. 2000, pp. 2124. 